\title{
Un análisis a las teorías crimino-ambientales bajo la incidencia delictiva en García, Nuevo León
}

\section{An analysis of the crimino-environmental theories under the crime incidence in García, Nuevo León}

Juan Antonio Caballero Delgadillo*

Leonardo David Arriaga Avalos **

Octavio Quintero Ávila***

\section{Resumen}

El presente trabajo de investigación se realizó con el propósito de explorar y comparar algunas de las principales teorías de la corriente ambiental de la Criminología que estudian la relación de los factores ambientales o estructurales y la criminalidad, haciendo uso del análisis criminal o delictivo como herramienta de procesamiento de datos, y de esa forma sean aplicados en casos reales derivados de reportes del sistema de emergencia 9-1-1. La criminología ambiental es soportada por distintas teorías como: teoría del patrón delictivo, teoría de las actividades rutinarias, teoría de la elección racional y el triángulo de la criminalidad, que en términos generales hacen estudio de los elementos

Cómo citar

Caballero Delgadillo, J. A. Un análisis a las teorías crimino-ambientales bajo la incidencia delictiva en García, Nuevo León. Constructos Criminológicos, 2(2). Recuperado a partir de https:// constructoscriminologicos.uanl.mx/index.php/cc/ article/view/13

*https://orcid.org/0000-0001-9439-5696

Universidad Autónoma de Nuevo León

**https://orcid.org/0000-0002-5356-9116

***https://orcid.org/0000-0003-3922-9964
Recibido: 30-10-2021

Aceptado: 13-01-2022 involucrados en las conductas delictivas como: el delito, la víctima, infractor tiempo y espacio, criminalidad y rompimiento de una ley. El objetivo de este estudio es comparar en términos generales las teorías criminoambientales con incidencia delictiva y problemáticas sociales registradas en el sistema de emergencia del municipio de García, Nuevo León. Para el análisis se ha utilizado la teoría del patrón delictivo, teoría de las actividades rutinarias y la misma criminología ambiental para comprender si por medio del análisis de datos y uso de herramientas como mapas delictivos se puede aplicar las teorías en esta zona geográfica. Para cumplir con el objetivo se han utilizados distintas herramientas como el Paquete estadístico para las ciencias sociales, la herramienta Excel y Google Maps.

Palabras clave: Criminología, Criminología ambiental, análisis delictivo, patrón delictivo

\section{Abstract}

This research work was carried out with the purpose of exploring and comparing some of the main theories of the Environmental Criminology that study the relationship between environmental or structural factors and criminality, making use of criminal analysis as a 
data processing tool, and thus be applied in real cases derived from reports of the 9-1-1 emergency system. Environmental criminology is supported by different theories such as: criminal pattern theory, routine activities theory, rational choice theory and the triangle of criminality theory, which in general terms study the elements involved in criminal behaviors such as: crime, the victim, the offender, the time and space, criminality and law infringements. The objective of this study is to compare in general terms the environmental criminology theories with criminal incidence and social problems registered in the emergency system of the municipality of García, Nuevo León. For the analysis, the theory of the criminal pattern, the theory of routine activities and the environmental criminology have been used to understand whether the analysis of data and use of tools such as criminal maps, can be applied in this particular geographical area. To meet the objective, different tools have been used such as Statistical Package for the Social Sciences, Microsoft Excel and Google Maps.

Key words: Police, Pandemic, Rights' Restrictions

\section{INTRODUCCIÓN}

La criminología ambiental tiene como antecedente investigaciones dondeserelacionan cifras estadísticas, el uso de mapas para identificar de forma gráfica la concentración del delito y la influencia de elementos ambientales que aumentan las probabilidades de comisión de delitos. No es hasta a partir de la década de los 70 cuando los principales autores y referentes hacen aparición para ser los pilares en de esta corriente en las últimas décadas para este caso esta Oscar Newman con su trabajo de espacios defendibles y Ray Jeffery con su metodología CPTED o prevención del delito mediante el diseño urbano y antes de ellos se puede relacionar la investigación de Jane Jacobs "Muerte y vida de las grandes cuidades", que dejan a relieve la pregunta de ison aplicables algunas de las teorías de la criminología ambiental en el contexto mexicano?, para este caso en la municipalidad de García en el Estado de Nuevo León. La recopilación de información de distintos autores como (Boba, 2017), (Bruce C. W., 2017a),(Soto, 2016 b) y (Bruce W. C., 2010b) las teorias con mayor presencia en la criminologia ambiental son: teoria del patrón delictivo, teoria de las actividades rutinarias, teoria de la elección racional y el traingulo del delito y la vez son auxiliadas por el analisis de datos criminales, pero tambien se puede agregar la teria de los Circulos de David Canter y el perfil geografico de Kim Rossmo y Summer.

Sin duda es necesario realizar investigaciones basadas en la ciencia que puedan apoyar a prevenir la criminalidad, y buscar basar las acciones o estrategias en estos estudios elevando la participación de la ciencia y la practica policia en la prevención del criminalidad por medio del analisis criminal y la criminoligia ambiental.

\section{MARCO TEÓRICO}

\section{¿Qué es la criminología ambiental?}

La criminología ambiental puede ser confundida por las palabras que conforman su concepto, en específico aquella que trae confusión es "ambiental" y se puede relacionar con delitos ecológicos, tema donde algunos juristas pueden intervenir, para eso es necesario establecer algunos antecedentes y su misma definición. Continuando con el análisis del párrafo 
anterior, la Criminología ambiental, no siempre es comprendida de la mejor manera, no todos pueden entender a los que se refiere, por su parte (Soto, 2015), menciona que: la Criminología ambiental no hace referencia a los eventos delictivos ambientales, ni tampoco hace referencia a los delitos ecológicos. Las palabras pueden confundir a aquellos ajenos de la criminología, pensando que las legislaciones ambientales son el punto central para estudiar tema que los criminólogos deben de dejar claro.

Esta corriente de la criminología fue dada a conocer por los esposos Paul y Patricia Brantingham en la década de 1980 (Bruce, 2017a). Principalmente la concentración del desarrollo criminológico de esta corriente se centra en estudiar como el entorno influye y participa en aumentar y disminuir las posibilidades de ocurrencia de un crimen.

Brantingham \& Brantingham (1991) en su obra Criminología Ambiental en la parte de introducción: las dimensiones del crimen señalan que:

"La Criminología Ambiental plantea que los eventos delictivos deben de entenderse como una interacción de infractores, victimas u objetivos del delito y normativas legales, en escenarios específicos, ocurriendo en un momento y lugar específico" citado de (Vozmediano \& San Juan, 2010).

Desde este punto de vista "explicación y análisis ambiental del delito" en necesario tener presentes cuatro componentes del delito: el infractor o delincuente, victima u objetivo, aspecto legal y los elementos espacio- temporales.
Las investigaciones relacionadas a la corriente ambiental ponen en relieve la evolución y adaptación a las formas de investigación y explicación del delito, precisamente esto es comprobado con el uso de llamado "mapeo delictivo" y el uso de datos criminales como fuentes de información que por medio de su procesamiento buscan ser distribuidos a quienes los necesitan para tomar decisiones y combatir el delito a este último elemento se le llama base de datos o almacenamiento de datos.

En primera parte la "georreferenciación del delito" entendiéndose por este término como acción de hacer referencia al análisis y ubicación del delito. Para poder realizar este tipo de actividad es necesario utilizar herramientas como el Sistemas de Información Geográficos (SIG), ya que permite ubicar de manera precisa diferentes hechos sociales en específico para este caso eventos relacionados a la criminalidad.

Los mapas del delito son el producto de utilizar los programas donde puede ubicar eventos sociales (SIG), (Hein, 2005) señala que los mapas ayudan a describir de forma gráfica la distribución de distintas variables de la criminalidad como concentración y distribución de las conductas delictivas y su relación con las características situacionales y sociales.

Precisamente este punto, da comienzo a el uso de las técnicas de localización de los delitos reportados, que son organizados y procesados por medio de bases de datos o almacenamiento de información criminal, donde interviene directamente el análisis delictivo o criminal, parte práctica de la investigación. 
El almacenamiento de datos criminales puede ser representado como las técnicas cuantitativas del delito en específicos las estadísticas policiales que son aquellas que recolectan las denuncias, delitos, reportes de toda una zona o sectores específicos (USAID, 2015). Estas fuentes de información no son únicas de los temas policiales, pero depende del acceso que se pueda tener, por lo cual el análisis delictivo se basa en datos policiales principalmente dejando espacio para datos sociales en caso de tener acceso a ellos.

Así, mismo la búsqueda de temas sobre la criminología ambiental trae consigo distintas actividades, que facilitan la explicación de su objeto de estudio y su relevancia con el estudio al delito, para esto es necesario de varios temas como: mapas del delito, teorías explicativas del delito espaciales, análisis delictivo, factores ambientales y datos involucrados en el delito como víctima, infractor, guardián, lugar y criminalidad.

\section{Teorías espaciales explicativas del delito}

En la criminología existen distintas formas de explicación de la ocurrencia del delito para autores como (Rodriguez, 2012) existen múltiples direccione como: dirección clínica, dirección critica, dirección sociológica, dirección biológica, dirección psicológica y una más que inicio con el estudio de la criminología, la dirección antropológica.

Por otro lado, para el autor del libro Tratado de Criminología (Pablos de Molina, 2003) la agrupación por modelos teóricos, el primero es para el modelo biológico, modelo psicopatológico (psiquiátrico, el psicodinámico (psicoanálisis) y el psicológico, modelo de teorías sociológicas pluri factoriales, escuela de chicago y teorías ambientalistas, estructural funcionalista, teorías subculturales, teorías del proceso social y teorías del control social, las teorías del procesos social (labeling approach), y los modelos conflictuales.

Teniendo en cuenta los comentarios anteriores, es necesario establecer que las teorías que explican el delito desde esta corriente en la Criminología, analizan la asociación entre el contexto espacial y ambiental, en la misma ocurrencia del crimen, e interpreta las interacciones entre las variables ambientales que pueden facilitar o inhibir la comisión de un delito, así mismo también se habla del estudio del delito, criminalidad y victimización en lugar y tiempo determinados (E \& Wiles, 2014) y (Norza, Vargas, Avendaño, Rincón, \& Ospino, 2017).

Continuando con el tema las principales teorías e investigaciones relacionadas a la influencia del ambiente como elemento que aumenta las posibilidades de comisión del delito están: teorías de las actividades rutinarias, teorías de la elección racional, teoría del patrón delictivo y el triángulo de la criminalidad.

\section{Teoría de las Actividades rutinarias}

La teoría de las actividades rutinarias fue desarrollada en 1979 por los investigadores Marcus Felson y Lawrece E. Cohen, la principal explicación de esta teoría, es que en la delincuencia, coexisten tres elementos que conforman al delito en tiempo y espacio determinado, la víctima, el infractor motivado 
y la ausencia de un guardián, y que a la vez de manera individual, afectan directamente de manera negativa o positiva según sea su participación en el delito, esto puede derivar en haber un aplazamiento, cancelación u aumento de la tasa de delitos sin importar las condiciones sociales (Andersen, 2020) y (Soto, 2016).

Para ser más específicos la "Teoría de las actividades rutinarias" Cohen y Felson plantean que los incidentes violentos y la victimización, aumenta cuando un posible infractor se encuentre motivado, donde él mismo debe de converger en el tiempo y espacio adecuando junto con la victima potencial, en ausencia de aquellos mecanismos de seguridad ya sean formales o informales (Fundación Paz Cuidadana; Internacional Association of crime analysts; Motorola Solutions Foundation, 2016). Llegados al punto de resumir esta teoría crimino-ambiental, según Wortley y Mazerolle, 2008, citado de (Jiménez, 2012), el delito ocurre cuando los tres elementos principales que lo componen, infractor, victima u objetico y ausencia de guardián colisionan en el mismo tiempo y espacio determinado, pero que si el objetivo y el guardián tiene un cambio puede influenciar en la conducta del infractor evitándolo.

La teoría plantea que existen distintos actores involucrados en la comisión de conductas delictivas en especia aquellas de oportunidad, donde el elemento activo "infractor" tiene contacto con una posible victima u objetivo y también se tiene que señalar que él hace uso del conocimiento adquirido por vivencias y traslados realizados en zonas específicas, pero para que se pueda cumplir esta parte debe de estar ausente el elemento de protección o guardián, como un breve ejemplo, las actividades que desempeñan las personas están basadas en tiempo y lugares específicos, como horarios de entrada y salida en trabajo y escuelas, horarios establecidos para tomar alimentos, mismo horarias que generan rutinas y generan oportunidades para los infractores como partes del día y lugares con poca vigilancia formal por policías y natural por la misma comunidad o la víctima, dando la apertura para la comisión de conductas delictivas.

\section{Teoría del Patrón delictivo}

Patricia L. Brantingham y Paul J. Brantingham fueron los que propusieron la integración de las teorías del ambiente físico y la motivación del delincuente y de esta forma nació la teoría del patrón delictivo, en la cual se necesita la presencia de un delincuente, en segunda parte se debe de haber actividades rutinarias que realiza el delincuente, su vida diaria es la que le ofrecerá las oportunidades para las conductas delictivas, la tercera condición es la aparición de un suceso desencadenante como un objetivo disponible, pero todos esto está determinado por un esquema que se forma el infractor en su mente como resultados de actividades realizadas cotidianamente (Andersen, 2020) y (Soto, 2016a).

Hay que mencionar además que según (Brantingham \& Brantingham, 1984) esta teoría es una composición de más teorías enfocadas al ambiente físico y la motivación del delincuente, como ya se mencionó la teoría fue creada por este matrimonio, ellos siguieren que las conductas delictivas acontecerán con mayor posibilidad 
cuando la conciencia espacial del infractor se cruce con el objetivo ambicionado y con un bajo nivel de riesgo para él. Lo que lleva a mencionar que los criminales, por ende, no eligen de forma azarosa el lugar donde cometerán el delito, aunque la victima si puede ser cualquiera, los infractores son conscientes de la situación espacial donde elegirán a su objetivo.

La teoría del patrón delictivo hace referencia a que los delitos están determinados por oportunidades, actividades diarias y motivación del infractor que dan pie al conocimiento de las zonas con mayor vulnerabilidad y acceso a posibles víctimas, determinado que existen lugares con mayor concentración de conductas delictivas, pero no limitándonos a esto también existen horarios, infractores y tipos de delitos que dominan la estadística criminal.

La investigación cobra relevancia al identificar por medio de base de datos de registros de emergencia del sistema 9-1-1, el dominio de estas características relacionadas al delito, donde más adelante serán tratadas y explicadas.

\section{Teoría de la Elección racional}

Las principales teorías que sustenta las explicaciones de las conductas delictivas bajo la corriente ambiental de la criminología están relacionadas una con otras y para poder entender a la criminología ambiental es necesario conocerlas a profundidad, pero cada una de ellas tiene una explicación distinta de la ocurrencia de los eventos delictivos.

Por su parte la teoría de la elección racional fue desarrollada por Cornish y Clarke en
1985 y 1986. Esta teoría junto con la teoría de actividades rutinarias tiene gran influencia en la prevención de la delincuencia y la criminología, estas teorías se complementan, la segunda también conocida como TAR, es una macro- teoría que relaciona a la criminalidad con las actividades de la sociedad; en el caso de la primera y conocida también como TER, es una micro- teoría basada principalmente en el análisis que hacen los delincuentes sobre las oportunidades para cometer delitos (Andersen, 2020) y (Soto, La prevención situacional: Bases teóricas de fundamento criminológico, 2016).

Los infractores prácticamente buscan maximizar su placer y minimizar el dolor, y por lo tanto toman la decisión de delinquir cuando el riesgo de ser detenidos es menor a las potenciales recompensas, lo que significa que el raciocinio en el delito está presente antes y después de cometer la conducta delictiva (Taylor y Harrell, 1996) citado de (Paulsen, Bair, \& Helms, 2012). Contario a lo que se puede pensar sobre algunos infractores esta teoría hace referencia a la importancia que tiene la toma de decisiones basada en un proceso que tiene el posible infractor antes de realizar una conducta, la cual le puede beneficiar, pero que principalmente, toma la decisión basada en el conocimiento previo basado en las consecuencias, y en un análisis espacial que le permita librarse del lugar y después de una posible sanción de un guardián formal o informal.

\section{Triangulo del delito}

Esta herramienta nace de las principales teorías de la criminología ambiental, en especial influenciada de la teoría de las actividades 
rutinarias. El triángulo de la criminalidad o del problema como también se conoce, está compuesto por dos partes, una interna y otra externa de las cuales cada uno tiene distintos componentes y funciones que dan explicación a la ocurrencia del delito y sus posibles soluciones.

Triangulo interno (Clake \& Eck. John, 2014):

Victima/Objetivo: En este caso también se le conoce como blanco debido a que en este elemento recae el daño de la acción delictiva. Existe una distinción importante entre la víctima y el objetivo, en el caso de la víctima se ve afectado una persona, por el contrario, el robo o sustracción de un objeto representa al objetivo. Este elemento tiene varias características importantes como ser valioso, atractivo, accesible y de fácil sustracción.

Futuro Infractor o delincuente: Este componente está representado por una persona que se encuentra dispuesta a cometer un delito o conducta antisocial. Es relevante mencionar que, si las características espaciales son propicias, ayudarán a tomar la decisión a sopesar si la recompensa es mayor al esfuerzo o riesgo de las sanciones que podrá conseguir.

Lugar: está relacionado al contexto o características del espacio físico urbano, como bares, antros, cines, teatros mismos que representan el factor económico, también pueden ser parques o centro de la cuidad como contexto social y cultural, donde puede cometerse la conducta delictiva o antisocial
Triángulo externo: (Sepúlveda, 2010) y (Clarke \& John, 2003):

Vigilante o guardián: este elemento puede ser ocupado por personas desde dos aspectos, en primera parte por aquellos que realizan acciones de autoprotección, como resguardar su patrimonio, familia, compañeros o amigos, en segunda parte están los individuos que tiene aquel rol formal como un guardia de seguridad, policía o personas que ayuden a disuadir al delito.

Controlador: este individuo tiene una relación cercana con el posible infractor del delito, debido a que se encuentra en una posición que le permite ejercer presión y control de las acciones, como un padre, catedrático, amigos e incluso esposa o hijos.

Responsable: el administrador del lugar ejerce control sobre este espacio, esta persona puede ser, el dueño del lugar, un profesor o una persona que es la encargada de vigilar como un guardia.

Este apartado teórico menciona las principales teorías que se pueden encontrar para dar explicación al delito desde una perspectiva ambiental, pero bajo investigaciones se pueden agregar también las siguientes:

\section{Prevención situacional del delito}

Prevención situacional del delito que consiste en modificar el entorno para propiciar la convivencia y la cohesión social, así como disminuir los factores de riesgo que facilitan fenómenos de violencia y de incidencia 
delictiva, mediante algunos puntos específicos como lo son: el mejoramiento del desarrollo urbano, ruta, ambiental y diseño industrial, el uso de nuevas tecnologías, la vigilancia con respeto a la intimidad de las personas y algunas estrategias para garantizar la no repetición de casos de victimización (Ley General para la Prevención Social de la Violencia y la Delincuencia, 2012).

\section{Círculos de David Canter}

La Teoría de los círculos de David Canter, toma en cuenta la ley del costo-beneficio y la teoría de las actividades rutinarias, asumiendo que todos los delincuentes tienen una base de operaciones o también conocidos como punto de anclaje, esto deja al alcance que se puede realizar un análisis geográfico de sus crímenes, relacionando las conductas delictivas con el infractor (Jiménez, 2012).

Esta teoría fue propuesta por David Canter y Larkin en 1993, que como en el párrafo anterior se señala, proponen que existe un lugar donde se cometen los delitos, relacionado con el posible espacio donde operan los delincuentes. De esta forma David y Larkin comprobaron, que mediante el dibujo de un círculo cuyo diámetro fuera la distancia entre los dos delitos más alejados del agresor serial, podría establecerse el lugar donde habita el agresor. Se realizó un estudio donde se analizaba a 45 violadores seriales y comprobaron que el $87 \%$ de ellos vivían dentro de este círculo (Canter \& Donna, 2008).

El modelo de Canter es bajo en costos, a comparación del modelo predictivo de Kim Rossmo Ilamado Criminal Geographic Target, que busca la probabilidad de ocurrencia del delito en una zona por medio de softwares, por lo contrario, Canter como ya se mencionaba traza círculos que tocan el diámetro de los delitos más distantes. Así el autor de esta teoría sostiene que el lugar de operaciones y el lugar del delito casi siempre estarán dentro del círculo, tamaño que va aumentando conforme cometen más crímenes (Suárez \& Chías, 2015).

\section{Análisis delictivo o análisis criminal}

El análisis delictivo puede ser confundido debido a que la conformación de este concepto utiliza palabras que pueden ser comprendidas como acciones de interpretación general en específico como el análisis sin profundidad y realizado por cualquier individuo, pero al identificar la noción como un proceso especifico de sistematización de datos llevado a cabo como una profesión aumenta su valor y se visualiza su complejidad.

Para (Boba, 2017) el análisis criminal tiene una concepción más amplia que otros investigadores en este tema:

El análisis delictivo analiza más que el delito, incluido muchos tipos de información que son relevantes para la policía, como desordenes, llamadas de servicios, problemas de calidad de vida, accidentes de tránsito, incidentes críticos y con menos frecuencia incendios e incidentes médicos de emergencia.

Complementado el entendimiento del análisis delictivo o criminal, y bajo un enfoque donde es necesario el uso de datos de manera universal más allá de solo datos policiacos, existe un concepto 
que es utilizado por la International Association of Crime Analysis (IACA) y que en español es conocida como la Asociación Internacional de Análisis del delito de manera estándar:

Una profesión y un proceso en el que un conjunto de técnicas cuantitativas y cualitativas se utilizan para analizar datos valiosos para las agencias policiales y sus comunidades, incluye el análisis de delitos y crímenes, víctimas del crimen, el desorden, la calidad de vida, problemas de tráfico y operaciones internas de la policía y sus resultados apoyan a la investigación y persecución criminal, patrullajes, estrategias de prevención y reducción del crimen, resolución de problemas y la evaluación de los esfuerzos de la policía, citado de (Boba, 2017).

La conceptualización justifica su trabajo y el uso de los datos de manera sistemática como herramienta de la cual se puede hechas una mano y dar explicación a las conductas delictivas, o relacionar bajo su labor los datos procesados por esta profesión con la corriente ambiental de la Criminología.

La combinación o el trabajo donde se involucra el análisis delictivo y la criminología ambiental tiene como objeto de estudio varios participantes que bajo su análisis se convierten en datos los cuales son: victima, infractor, guardián, tiempo y lugar, criminalidad estos como elementos principales.

La principal herramienta utilizada en el análisis delictivo es el Ilamado Sistema de información geográfica que es la representación de la distribución de delitos y en segunda parte y que es menos conocida por la forma de la publicación son las bases de datos, que sirven como herramientas de trabajo a la criminología ambiental para explicar al delito.

\section{El comienzo de las investigaciones}

Las investigaciones a las que se les puede atribuir el comienzo la criminología ambiental son aquellas que fueron desarrolladas por André Guerry y Adolphe Quetelet en donde se utilizaron datos y mapas para la identificación de conductas delictivas combinado la estadística y los elementos ambientales.

El estudio de la distribución espacial del delito y la criminalidad se remonta al trabajo de Adolphe Quetelet y André Guerry, esto nacido en el siglo XIX (Andersen, 2020), que en contrastaste con el siglo XXI con el uso del sistemas operativos o computarizados, ya que los primeros estudios se trazaban mapas a mano, en el caso moderno, los datos son utilizados en grandes cantidades por el sistema seguridad ya sea pública o privada, para este caso son utilizados los Ilamados SIG'S) donde se generan mapas del delito.

Quetelet en 1842 realizó el primer trabajo, un análisis geográfico de manera estadística, estudiando la correspondencia entre incidencia delictiva y otras variables del análisis ecológico. Este trabajo lo realizó con el fin de conocer los factores que gobiernan las conductas antisociales, lo que lo llego a llegar a la hipótesis que en la actualidad se sigue conociendo sobre la estacionalidad criminal. A pesar de lo anterior, otras teorías han sido tomadas con mayor aprecio, como algunos 
estudios que hablan de la genética, sociología o aspectos mentales que son más conocidos o mencionados (Suárez \& Chías, 2015).

Es relevante mencionar que a más de 150 años de algunas de las primeras investigaciones relacionadas a la estacionalidad del delito utilizandoherramientascomomapasyestadística y como almacenamiento papel, lo podamos ver en el siglo XXI en usos computarizados bajos programas espaciales de almacenamiento $y$ representación, lo que demuestra que el delito cumple con características específicas y así como las conductas evolucionan las ciencia también utilizando la tecnología para explicar el comportamiento delictivo.

Existe una relación entre el análisis de información criminal y la criminología ambiental, no solo en temas prácticos como trabajo y procesamiento de datos sino como soporte una de otra, la relación entre el análisis criminal y la misma criminología ambiental se encuentra en las teorías criminológicas que sustentan el trabajo del análisis criminal, ya que las teorías ofrecen gran cantidad de información y el análisis delictivo la aprovecha para poder trabajar con ella y de esa forma obtener productos que ayuden a prevenir las conductas delictivas.

\section{METODOLOGÍA}

\section{Datos para la explicación y sustento de la criminología ambiental y metodología empleada}

Los datos nacen del Sistema nacional de emergencia 9-1-1 y fueron trabajados por medio de un análisis de (umbral) entendiéndose por este como aquel proceso donde se realiza el procesamiento de datos estadísticos a nivel básico.

El municipio de García del Estado de Nuevo León cuenta con una población total de 247,370 habitantes que representan el $4.83 \%$ de la población de Estado de Nuevo León.

Para relacionar el análisis de datos con construcciones teóricas de la criminología ambiental se eligió la colonia Valle de Lincoln que cuenta con múltiples sectores y según la metodología para el análisis de información se seleccionó de la misma colonia los sectores: San José, San Agustín y El Fraile, teniendo por objetivo identificar la incidencia delictiva con mayor cantidad de eventos bajos dos aspectos: Análisis delictivo y Criminología ambiental.

La metodología empleada está basada en dos puntos específicos la primera donde se expone el papel del análisis delictivo como proceso para identificar zonas de mayor concentración delictiva y la segunda el análisis delos constructos teóricos de la criminología ambiental teniendo como resultado la interacción de herramientas y ciencia.

El papel del análisis criminal bajo su categoría administrativa orienta y auxilia para la elección del área geográfica a trabajar, y que bajo una exhausta búsqueda en bases de datos de número de emergencia 9-1-1 y la indagación de reportes efectivos o confirmados registrados en los años 2014, 2015 y 2016 se obtuvo como resultado la Colonia Valle de Lincoln. 
En términos metodológicos la colonia Valle de Lincoln fue el área de mayor incidencia delictiva en el Municipio de García en un periodo de 2014, 2015 y 2016, según la base de datos del sistema de emergencia 9-1-1 y 066, dentro de los problemáticas registradas se encuentran delitos patrimoniales (robo a casa habitación, negocio, vehículo y a transeúnte con violencia y o sin violencia), así mismo se identificaron altos índices de eventos integrados al Kaizen social ( Consumo de alcohol en vía pública, consumo de alcohol en vía pública, ruido excesivo, personas agresivas, riña, violencia familiar, alteración del orden público por persona alcoholizada, alteración del orden público por persona drogada y asociación delictuosa o pandillerismo.

Con apoyo de la georreferenciación entendiéndose por esta como el uso de coordenadas en un mapa para asignar una ubicación espacial, se plasmaron áreas de riesgo, incidencia delictiva y problemas sociales ya mencionados.

La segunda parte basada en el análisis de constructos teóricos de la criminología ambiental está basada en la interpretación y relación de la teoría del patrón delictivo y explicaciones generales de la criminología ambiental, estableciendo una relación entre la teoría menciona y como se visualiza en términos prácticos.

\section{Patrón delictivo y Criminología ambiental en la práctica}

Los datos son importantes para que el análisis delictivo "parte práctica" pueda relacionarse con la teoría de corte ambiental, es por eso por lo que fue necesario la combinación de trabajos el primero basado en la elaboración de hotspot y mapas delictivos y la segunda identificación de información relevante en horarios y lugares específicos.

Figura 1. Esquema de operatividad

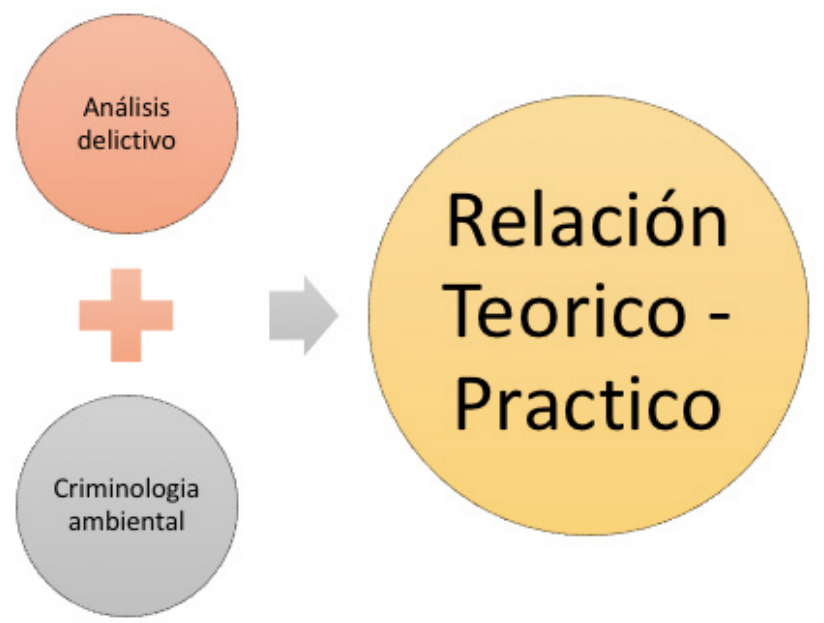

Dato: fuente esquema propio 


\section{RESULTADOS}

Graficas de distribución de problemáticas sociales

Tabla 1. Kaizen Social del año 2014.

\begin{tabular}{|c|c|c|c|c|c|}
\hline \multicolumn{6}{|c|}{ Kaizen Social del año 2014} \\
\hline & & Frecuencia & Porcentaje & $\begin{array}{c}\text { Porcentaje } \\
\text { válido }\end{array}$ & $\begin{array}{l}\text { Porcentaje } \\
\text { acumulado }\end{array}$ \\
\hline \multirow[t]{9}{*}{ Válido } & Enero & 37 & 6.9 & 6.9 & 6.9 \\
\hline & Febrero & 42 & 7.8 & 7.8 & 14.6 \\
\hline & Marzo & 75 & 13.9 & 13.9 & 28.5 \\
\hline & Abril & 63 & 11.7 & 11.7 & 40.2 \\
\hline & Mayo & 62 & 11.5 & 11.5 & 51.7 \\
\hline & Junio & 80 & 14.8 & 14.8 & 66.5 \\
\hline & Julio & 83 & 15.4 & 15.4 & 81.9 \\
\hline & Agosto & 98 & 18.1 & 18.1 & 100.0 \\
\hline & Total & 540 & 100.0 & 100.0 & \\
\hline
\end{tabular}

Elaboración Propia

Grafica 1. Kaizen Social del año 2014.

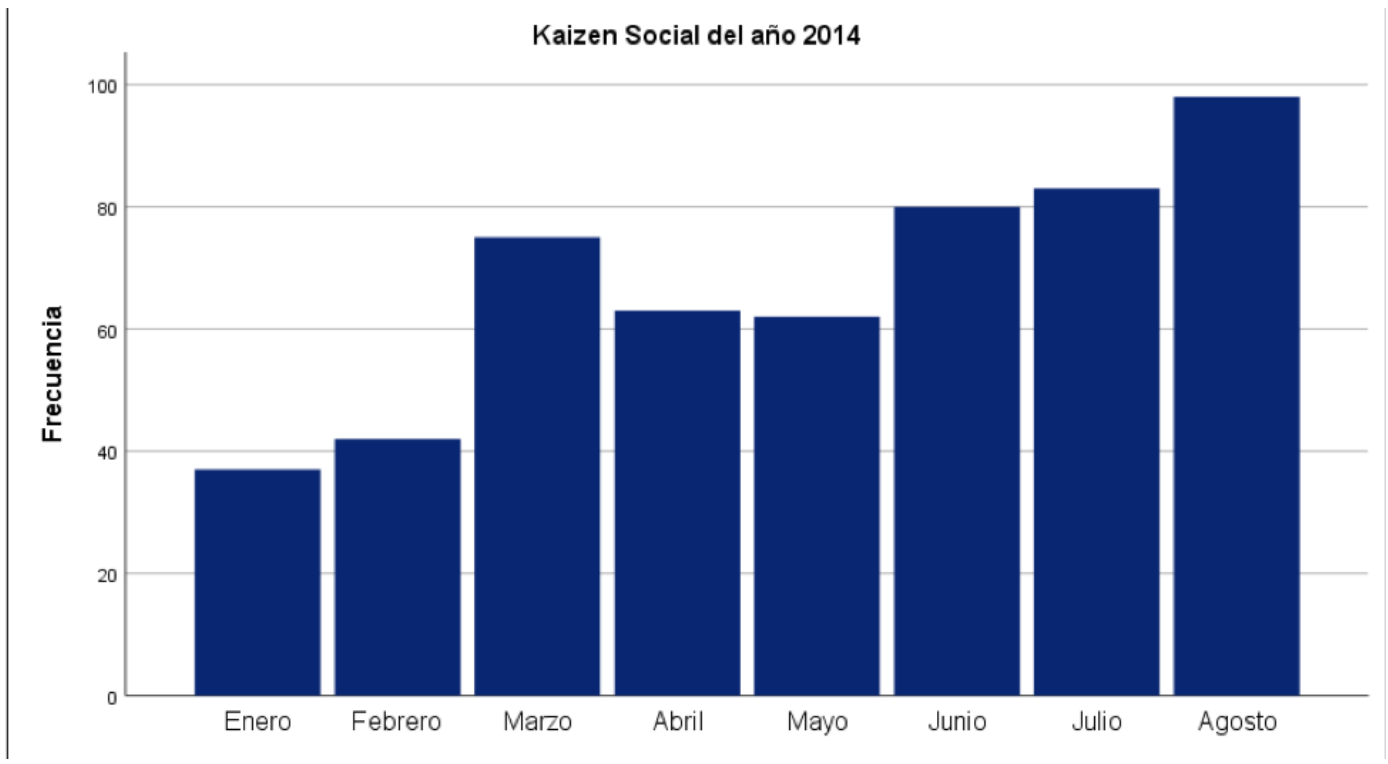

Elaboración Propia 
Tabla 2. Kaizen Social del año 2015.

\begin{tabular}{|c|c|c|c|c|c|}
\hline \multicolumn{6}{|c|}{ Kaizen social del año 2015} \\
\hline & & Frecuencia & Porcentaje & $\begin{array}{c}\text { Porcentaje } \\
\text { válido }\end{array}$ & $\begin{array}{l}\text { Porcentaje } \\
\text { acumulado }\end{array}$ \\
\hline \multirow[t]{9}{*}{ Válido } & Enero & 25 & 5.8 & 5.8 & 5.8 \\
\hline & Febrero & 37 & 8.5 & 8.5 & 14.3 \\
\hline & Marzo & 59 & 13.6 & 13.6 & 27.9 \\
\hline & Abril & 69 & 15.9 & 15.9 & 43.8 \\
\hline & Mayo & 64 & 14.7 & 14.7 & 58.5 \\
\hline & Junio & 57 & 13.1 & 13.1 & 71.7 \\
\hline & Julio & 67 & 15.4 & 15.4 & 87.1 \\
\hline & Agosto & 56 & 12.9 & 12.9 & 100.0 \\
\hline & Total & 434 & 100.0 & 100.0 & \\
\hline
\end{tabular}

Elaboración Propia

Grafica 2. Kaizen Social del año 2015.

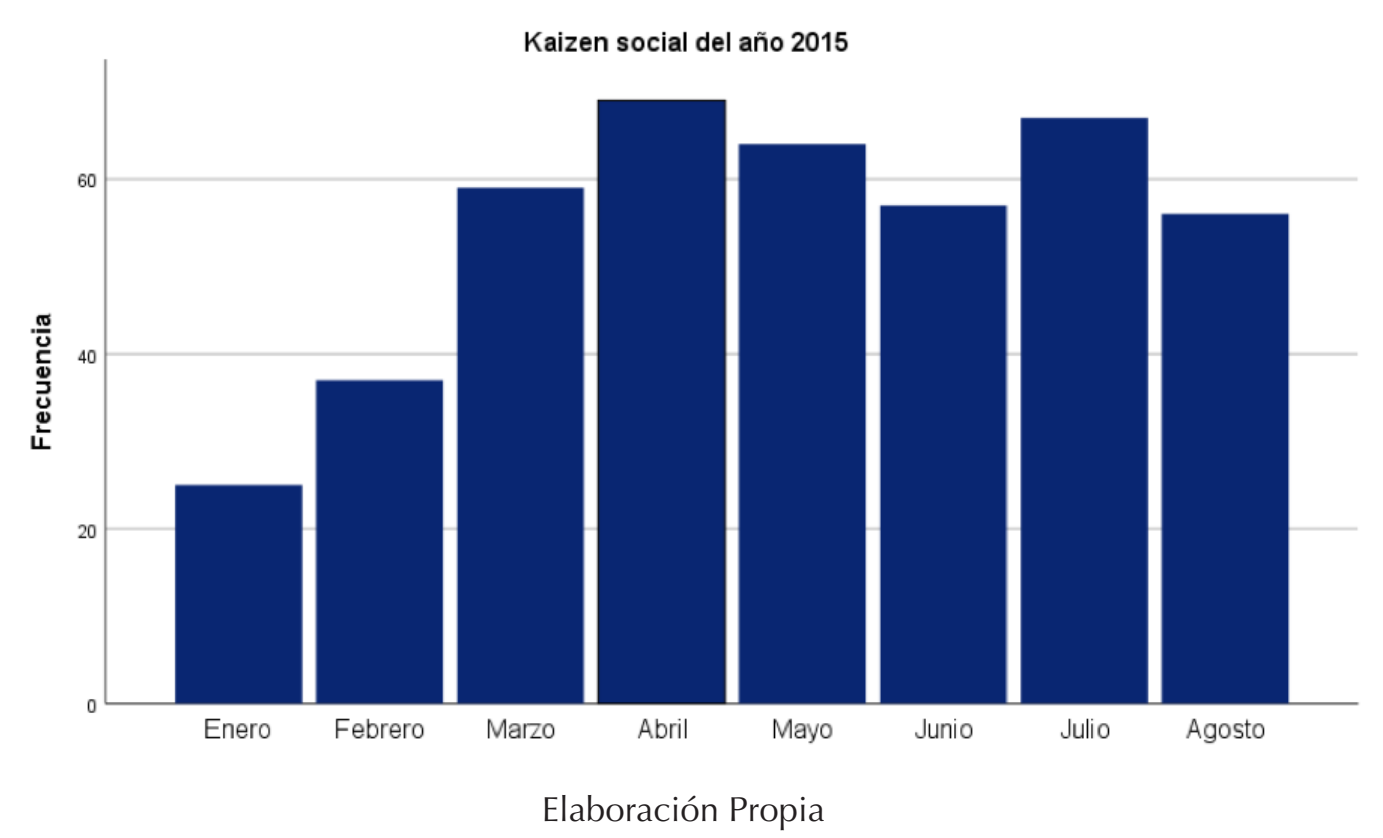


Tabla 3. Kaizen Social del año 2016.

\begin{tabular}{|c|c|c|c|c|c|}
\hline \multicolumn{6}{|c|}{ Kaizen social del año 2016} \\
\hline & & Frecuencia & Porcentaje & $\begin{array}{c}\text { Porcentaje } \\
\text { válido }\end{array}$ & $\begin{array}{l}\text { Porcentaje } \\
\text { acumulado }\end{array}$ \\
\hline \multirow[t]{9}{*}{ Válido } & Enero & 37 & 10.2 & 10.2 & 10.2 \\
\hline & Febrero & 28 & 7.7 & 7.7 & 18.0 \\
\hline & Marzo & 39 & 10.8 & 10.8 & 28.7 \\
\hline & Abril & 47 & 13.0 & 13.0 & 41.7 \\
\hline & Mayo & 58 & 16.0 & 16.0 & 57.7 \\
\hline & Junio & 57 & 15.7 & 15.7 & 73.5 \\
\hline & Julio & 52 & 14.4 & 14.4 & 87.8 \\
\hline & Agosto & 44 & 12.2 & 12.2 & 100.0 \\
\hline & Total & 362 & 100.0 & 100.0 & \\
\hline
\end{tabular}

Grafica 3. Kaizen Social del año 2016.

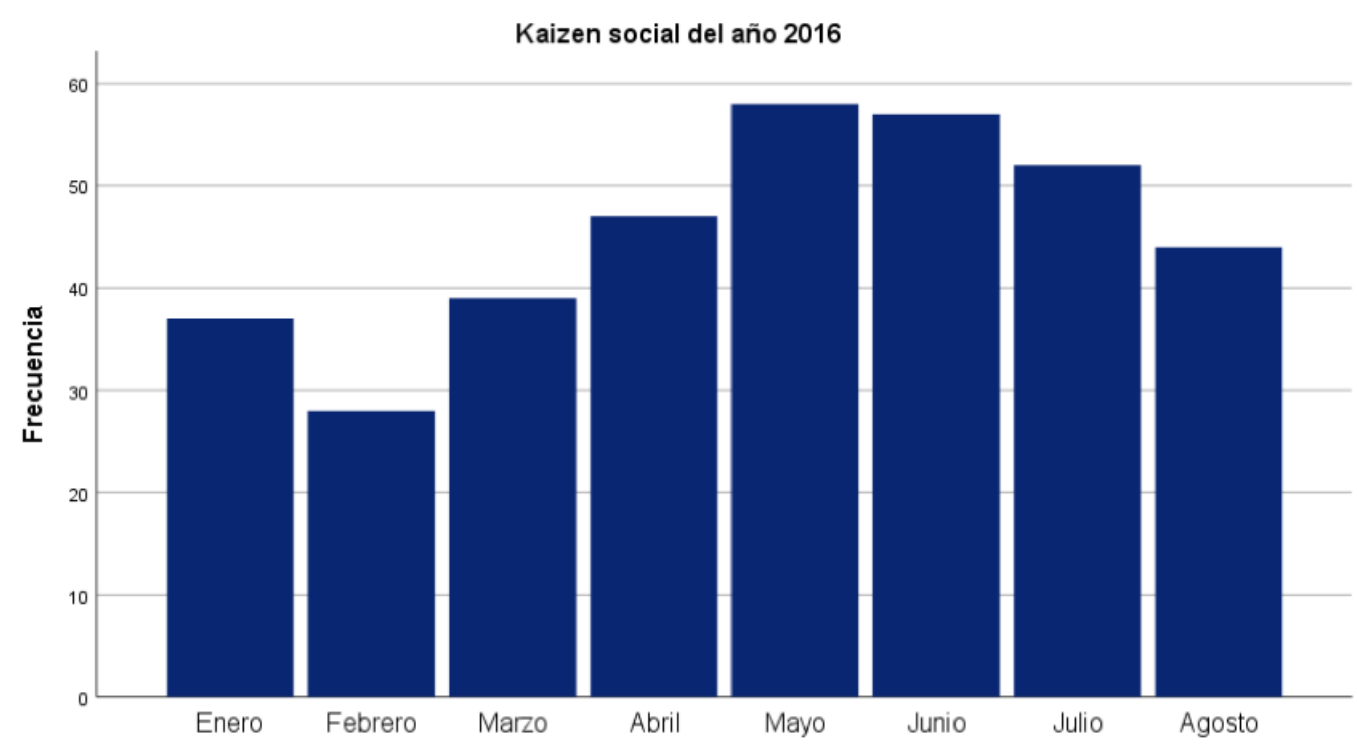

Elaboración Propia 
Grafica 4. Kaizen Social Periodo Enero agosto 2014 - 2017

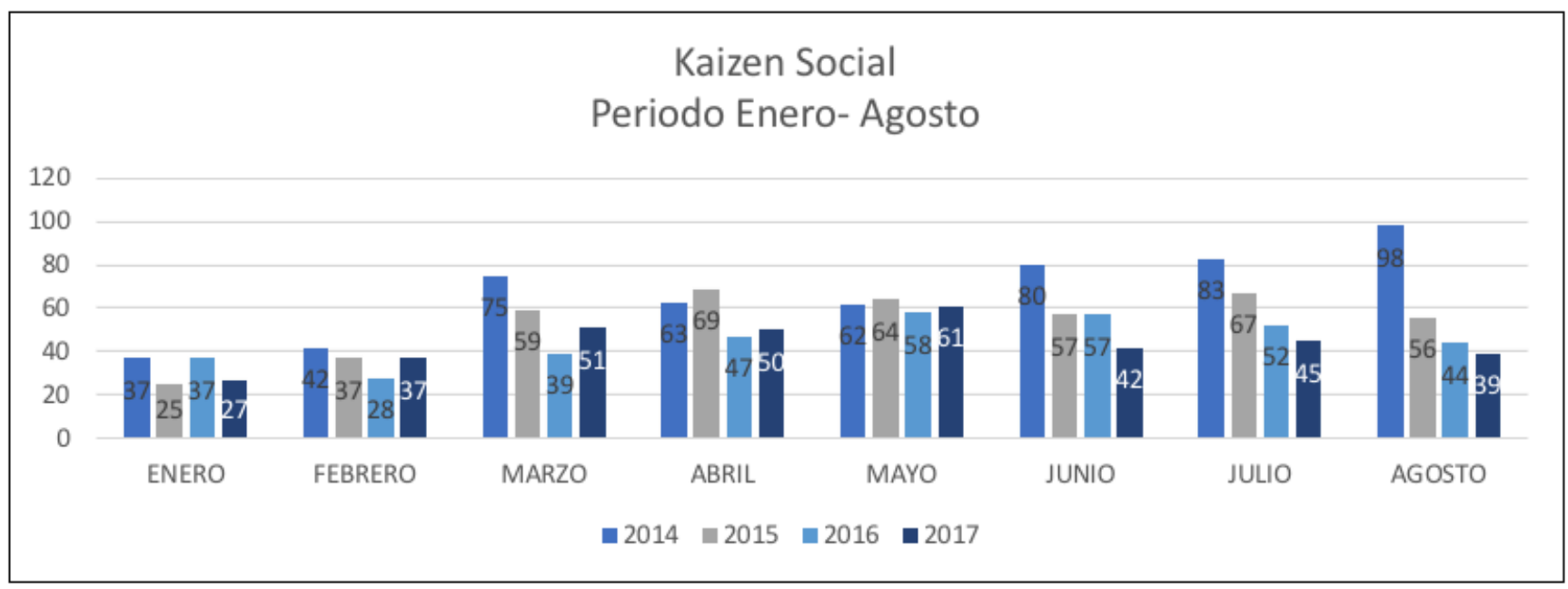

Elaboración Propia

Las conductas reportadas al sistema de emergencia conocidas como Kaisen social o entendida con otras palabras como problemáticas sociales, siguen un patrón de comportamiento derivado a las actividades rutinarias de personas que viven un espacio geográfico determinado, lo que significa que al observar la gráfica 4 general de incidencia delictiva de los años 2014, 2015, 2016 y 2017 existe una concentración estándar en el mes de mayo, demostrando que las conductas reportadas tiene un patrón temporal que ayuda a identificar los tiempo específicos, así mismo saber que cualquier modificación en las rutinas de una comunidad puede influir en el aumento de probabilidades de comisión de conductas delictivas.
Por otra parte, el aumento en los indicadores puede estar relacionado a la temporalidad de vacaciones de verano, vacaciones estipuladas que generan rutinas en la población como paseos fuera de la cuidad o mayor convivencia entre los miembros de una comunidad o la misma familia.

Continuado con el análisis los primeros meses del año reafirman la concentración temporal de los reportes de conductas delictivas, y que en términos generales adaptándose a la teoría del patrón delictivo que nos habla que la concentración de la criminalidad en lugares y tiempo específicos.

Tabla 4 Incidencia por mes y año

\begin{tabular}{c|c|c|c|c|}
\cline { 2 - 5 } & $\mathbf{2 0 1 4}$ & $\mathbf{2 0 1 5}$ & $\mathbf{2 0 1 6}$ & $\mathbf{2 0 1 7}$ \\
\hline \multirow{2}{*}{$\begin{array}{c}\text { Enero } \\
\text { Febrero }\end{array}$} & 37 & 25 & 37 & 27 \\
\cline { 2 - 5 } & 42 & 37 & 28 & 37 \\
\hline
\end{tabular}




\begin{tabular}{|c|c|c|c|c|}
\hline Marzo & 75 & 59 & 39 & 51 \\
\hline Abril & 63 & 69 & 47 & 50 \\
\hline Mayo & 62 & 64 & 58 & 61 \\
\hline Junio & 80 & 57 & 57 & 42 \\
\hline Julio & 83 & 67 & 52 & 45 \\
\hline Agosto & 98 & 56 & 44 & 39 \\
\hline Total & 540 & 434 & 362 & 352 \\
\hline
\end{tabular}

Elaboración Propia

La tabla 4 representa la concentración de la incidencia general de los años y mes de análisis, representando por medio de colores menor concentración en color verde, media en color amarillo y rojo en mayor.
Las conductas reportadas tienen aumentan conforme los meses del año van pasando, mostrando concentración después del cuarto mes del año y concentrándose en los meses de mayo, junio y julio.

Tabla 5 Resultados de marcha exploratoria de seguridad y áreas de riesgo

\begin{tabular}{c|c} 
Áreas de riesgo & Total \\
Basura & 38 \\
Casa Abandonada & 289 \\
Graffiti & 100 \\
Hierba Crecida & 7 \\
Terreno Baldío & 10 \\
Total & 444
\end{tabular}

Elaboración Propia

La influencia de los elementos ambientales o estructurales en una comunidad entendiendo para este análisis como una colonia o un municipio pueden afectar en el aumento de la criminalidad, mismo que es representados por la tabla 5, la criminología ambiental establece esta relación y se ve reflejado con los siguientes mapas. 
Imagen 1 Mapas de concentración delictiva de sectores y patrón delictivo

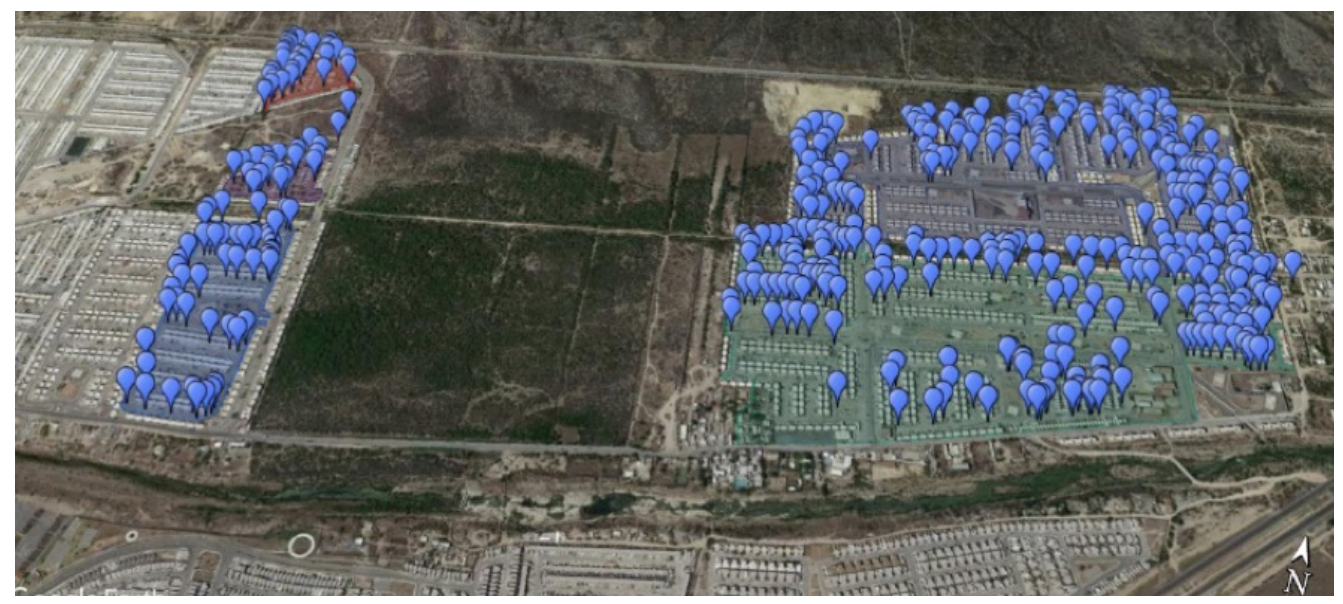

Elaboración Propia

Imagen 2 mapa de concentración delictiva de sectores y patrón delictivo hotspot

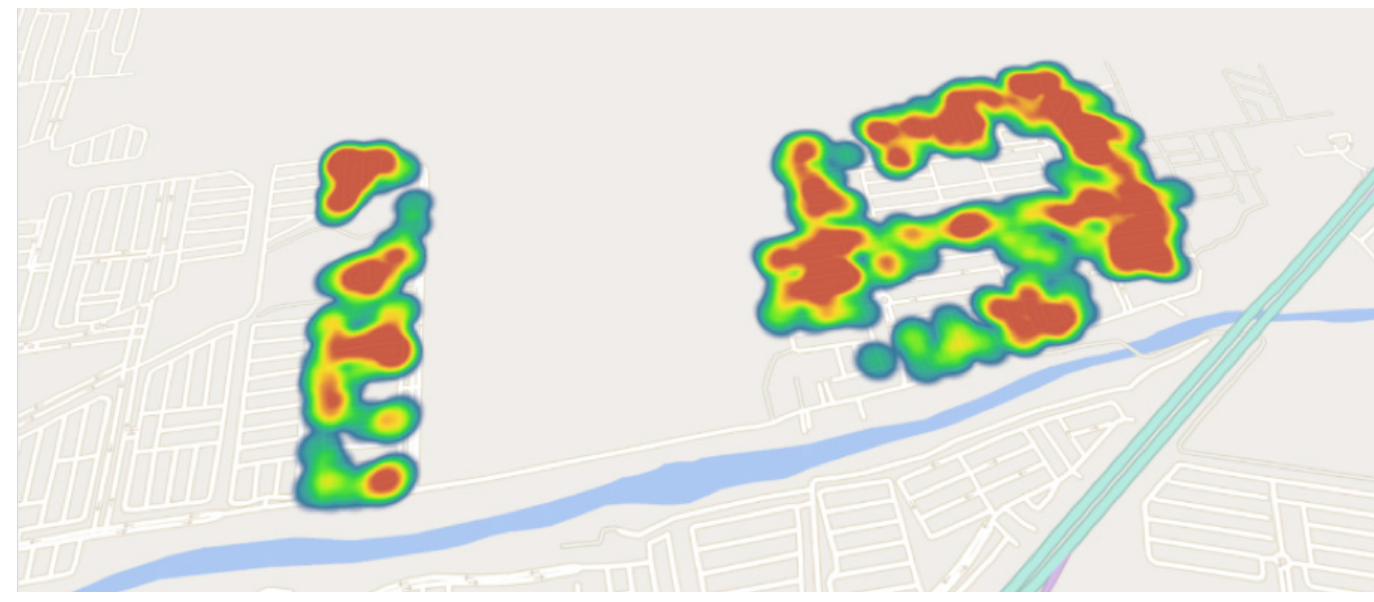

Elaboración Propia

Los mapas demuestran que las conductas delictivas no se concentran en toda la comunidad o área geográfica, sino que se focaliza o en zonas específicas, el mismo mapa orienta a identificar las zonas específicas que para este caso la periferia de la colonia es aquellas de mayor concentración de criminalidad.

EL uso de SIG's aporta información visual valiosa para poder determinar acciones preventivas por medio del procesamiento de datos Ilevadas de la mano de análisis delictiva o acciones disuasivas por elementos de seguridad encargados de la prevención del delito.

\section{Pasos para la recolección de datos e información:}

La metodología empleada para la recolección de información fue extraída de la base de datos del número de emergencia 9-1-1. 
El proceso de extracción de información tiene 4 pasos:

- Se realiza un filtro en para las colonias de mayor incidencia delictiva en términos de reportes en delitos patrimoniales (robo a casa habitación, robo a negocio, robo a transeúnte y robo a vehículo y problemas que pertenecen al Keizen social en el en el municipio de García, Nuevo León.

- Es elegida la colonia Valle de Lincoln y se sectoriza el área obteniendo mayor incidencia los sectores: Fraile, San José y San Agustín.

- Se trabaja de forma individual toda la colonia, y se obtuvieron horarios de mayor incidencia, la parte del día con mayor recurrencia días, partes de la semana y calles.

- De los datos obtenidos se obtuvo gráficas y tablas de horarios, lugares para cada sector.

\section{Interpretación de los resultados de la investigación}

La teoría del patrón delictivo nos ayuda a identificar en términos generales que la criminalidad o los delitos de manera individual no se comenten al azar sino que existen áreas de mayor concentración, tiempo, horarios, infractores y victimas que tienen mayor probabilidad de presentarse y todo esto puede estar influenciado debido a las características ambientales o estructurales de una zona geográfica, en el caso de análisis de datos reafirma que el patrón del delito para el municipio de García Nuevo León cumple con los criterios generales de la teoría elaborado por los esposos Brantingham.
La teoría de las actividades rutinarias y la misma Criminología ambiental como corrientes teóricas sustentan el trabajo, bajo el mismo análisis de identificación de áreas de riesgo como problemas estructurales o problemas ambientales, estableciendo la relación entre la incidencia delictiva y problemas estructurales no atendidos dejando un área de oportunidad para buscar prevención de la criminalidad.

El procesamiento de datos por medio de análisis criminal con soporte teórico de la Criminología Ambiental se relaciona en productos para la prevención, el uso de datos para identificar probabilidades de ocurrencia de delitos, la criminología estudia gran cantidad de elementos participantes de la criminalidad y el análisis delictivo al procesarlos y utilizarlos auxilian a los tomadores de decisiones para comenzar con el proceso de prevención.

\section{CONCLUSIONES}

Las investigaciones en la criminología han sido utilizadas y desarrolladas para dar explicación de la conducta delictiva en específico es debido a eso que existen distintas corrientes, dirección o modelos explicativos en la ciencia Criminológica. Los estudios de la influencia de las características ambientales de la comisión de conductas delictivas y usos de herramientas para representar y recolectar datos delictivos son tan viejas como la criminología clínica representada por el padre de esta ciencia Cesar Lombroso que con obras como "Los criminales" que da explicación de características físicas de los individuos y su relación con las conductas delictivas, o la publicación de su obra más conocidas como Lúomo Delinquente 
en 1876 (Landecho, 2015). Actualmente se ha puesto énfasis al análisis de datos criminales como herramienta auxiliar para la prevención y persecución del delito, pero es necesario darle el sustento científico de la criminología ambienta, ya que las investigaciones y las buenas prácticas pueden avanzar de forma considerable si se basan en estudios que fueron comprobados evitando caer en errores que otros ya tuvieron. Las teorías de la criminología ambiental que, aunque sus inicios e investigaciones nacen en Inglaterra son aplicadas para conductas delictivas en municipalidades de México, pero es necesarios comprobar en distintos lugares y zonas del país para sustentar la investigación a nivel macro.

\section{TRABAJOS CITADOS}

Andersen, A. M. (2020). Environmental Criminology. Evolution, Theory and Practice. New York: Routledge. Taylor \& Froncis Gruop.

Boba, S. R. (2017). Crime Analysis with Crime Mapping. United States of America: SAGE Publications, Inc.

Brantingham, P., \& Brantingham, P. (1984). Patterns in Crime. Nueva York, Estados Unidos de América : Macmillan.

Bruce, C. W. (2017). Fundamentals of Crime Analysis: Crime Analysis Terms, Concepts, and Processes. En I. A. Analysis, Exploring Crime Analysis: Readings on Essential Skill (3rd ed., págs. 1-28). Overland Park, KS.

Bruce, W. C. (2010). Fundamentos del análisis delictual. En F. p. cuidadana, \& A. i. delictuales, Análisis delictual: enfoque y metodología para la reducción del delito (págs. 27-55). Santiago, Chile : Fundación paz cuidadana .

Canter, D., \& Donna, Y. (2008). Applications of Geographical Offender Profiling. Liverpool, Reino Unido: Ashgate Publishing Company. Obtenido de https://books.google.com.mx/ books?hl=es\&lr=\&id=F3Ha4Lk_zF4C\&oi=fnd\&pg=PR7\& $d q=$ Principles+of+Geographical+Profiling.+Hampshire:
+ Ashgate+Publishing +Limited.\&ots $=$ wKcihWh5ta\&sig=1jLshwn864X9T9kOXHz1 IJbpxY \#v=onepage\&q=Principles\%20 of\%20Geographical\%20Profiling

Clake, R. V., \& Eck. John, E. (2014). Análisis delictivo para la resolución de problemas. En 60 pequeños pasos. U.S. Departament of Justice.

Clarke, R., \& John, E. (2003). Cómo ser un Analista delictivo. En 55 pequeños pasos. (A. A. Durán, \& L. C. Medina, Trads.) Londes: Jill Dando Institute of Crime Science.

E, B. A., \& Wiles, P. (2014). Criminología Ambiental. En M. Maguire, R. Morgan, \& R. Reiner, Manual de Criminología (V. A. Aparicio, Trad., págs. 421-482). Cuidad de México: Oxfor. Fundación Paz Cuidadana; Internacional Association of crime analysts; Motorola Solutions Foundation. (2016). Manual de Análisis Criminal para Observatorios de Seguridad en Gobiernos Locales: Prevención del delito y solución de problemas. Santiago, Chile: Fundación Paz Cuidadana.

Hein, A. (2005). La Georreferenciación como herramienta para el diagnóstico de problemas de seguridad cuidadana en el ámbito local. Revista Paz Cuidadana, 22-27.

Jiménez, S. J. (2012). Manual práctico del perfil criminológico. Criminal Profiling (Segunda ed.). Valladolid, España : Lex Nova.

Landecho, V. C. (2015). La tipificación lombrosiana de delincuentes: trayectoria humana y doctrinal de Cesare Lombroso. Madrid: Universidad Complutense .

Ley General para la Prevención Social de la Violencia y la Delincuencia. (24 de Enero de 2012). Ley General Para la Prevención Social de la Violencia y la Delincuencia. Cuidad de México, México: Diario Oficial de la Federación. Recuperado el 20 de Mayo de 2020, de http://www. diputados.gob.mx/LeyesBiblio/pdf/LGPSVD.pdf

Norza, C. E., Vargas, E. N., Avendaño, P. B., Rincón, H., \& Ospino, M. A. (2017). Criminología ambiental y homicidio en la cuidad de Bogotá. Revista de Estudios Sociales(63), 55-71. doi:https://revistas.uniandes.edu.co/doi/10.7440/ res63.2018.05

Pablos de Molina, G. A. (2003). Tratado de Criminología. Valencia : Tirant lo Blanch.

Paulsen, D., Bair, S., \& Helms, D. (2012). Comprendiendo el 
comportamiento delictual. En F. P. Cuidana, M. S. Fundation, \& A. I. Delito, Análisis delictual: técnicas y metodologías para la reducción del delito (págs. 5-19). Santiago, Chile: Fundación Paz Cuidadana.

Rodriguez, M. L. (2012). Criminologia. Cuidad de México, México : Porrúa.

Soto, U. C. (2015). Las dos caras de la prevención situacional: El desplazamiento y la difusión de beneficios. Madrid : Universidad Nacional de Educación a Distancia .

Soto, U. C. (Diciembre de 2016). La prevención situacional: Bases teóricas de fundamento criminológico. Inter Criminis. Revista de Ciencias Penales(15), 127-153.

Suárez, M. T., \& Chías, B. L. (08 de 01 de 2015). CRIMINAL GEOGRAPHY AND SERIAL MURDER: THE CASE OF JUANA BARRAZA. Archivos de Criminología, Seguridad Privada y Criminalística, 21. Obtenido de https://www. researchgate.net/publication/277331659_Homicidio_ serial_y_geografia_el_caso_de_Juana_Barraza

USAID, A. d. (2015). Manual para la capacitación en materia de prevención social de la violencia y la delincencia. Cuidad de México: Agencia de los Estados Unidos para el desarrollo Internacional .

Vozmediano, S. L., \& San Juan, G. C. (2010). Ciminología ambiental. Ecología del delito y de la seguridad. Barcelona: Editorial UOC.

\section{Leonardo David Arriaga Avalos}

Afiliación: Universidad Autónoma de Nuevo León, México

Pasante Doctorado en Criminología por Universidad Autónoma de Nuevo León. Máster en Criminología por Universidad Autónoma de Nuevo León. Y licenciatura en criminología por la Universidad Humani Mundial. Catedrático en Licenciatura de la Facultad de Derecho y Criminología de la UANL. Exanalista delictivo del municipio de García Nuevo León.

\section{Octavio Quintero Ávila}

Afiliación: Universidad Autónoma de Nuevo León, México

Pasante Doctorado en Criminología por Universidad Autónoma de Nuevo León. Máster en Criminología por Universidad Autónoma de Nuevo León. Y licenciatura en criminología por Universidad Autónoma de Nuevo León. Catedrático en Licenciatura de la Facultad de Derecho y Criminología de la UANL.

\section{Juan Antonio Caballero Delgadillo}

Afiliación: Universidad Autónoma de Nuevo León, México.

Doctor en Derecho con orientación en Derecho Procesal, Máster en Criminología por la UANL y Licenciado en Criminología. Coordinador del Doctorado en Criminología. Catedrático en Licenciatura y Posgrado, de la Facultad de Derecho y Criminología de la UANL, Integrante de Sistema Nacional de Investigadores SNI I. Ex Sub-secretario de Administración Penitenciario en el Estado de Nuevo León, Ex Presidente de la comisión de Carrera Policial de la Secretaria de Seguridad Pública del Estado de Nuevo, Ex Director General de Inteligencia de la Secretaria de Seguridad Pública del Estado de Nuevo León. Actualmente Coordinador de Inteligencia Criminal y Estratégica del Centro de Investigación en Cibercriminalidad, Derecho Digital y Ciberseguridad de la Facultad de Derecho y Criminología. Correo de contacto: alfacoca123@gmail.com 